\title{
An optical delayline based on excitable microrings
}

\author{
Thomas Van Vaerenbergh ${ }^{1,2, *}$, Martin Fiers ${ }^{1,2}$, Joni Dambre ${ }^{3}$ and Peter Bienstman ${ }^{1,2}$ \\ ${ }^{1}$ Photonics Research Group, Department of Information Technology, Ghent University - imec, Ghent, Belgium \\ ${ }^{2}$ Center for Nano- and Biophotonics (NB-Photonics), Ghent University, Ghent, Belgium \\ ${ }^{3}$ Computer Systems Lab, Electronics and Information Systems, Ghent University - imec, Ghent, Belgium \\ *Email: thomas.vanvaerenbergh@intec.ugent.be
}

\begin{abstract}
We present in simulation a photonic neural circuit achieving a 200 ns spike delay, based on excitability in microrings. This type of delayline paves the way towards fully integrated optical spiking neural networks.
\end{abstract}

\section{INTRODUCTION}

In Spiking Neural Networks (SNNs) information is encoded in the timing of the excitations of the neurons. Amongst many other optical nonlinear components, such as InP Photonic Crystal $(\mathrm{PhC})$ nanocavities [1], [2], or semiconductor ring lasers [3], Silicon-OnInsulator (SOI) microrings show excitable behaviour. Consequently, they seem suited to be used as a basic building block of an optical SNN, in which the neurons are connected to each other using waveguides and splitters [4], [5]. However, to process time-dependent information, SNNs need the basic memory functionality delivered by the delays of their interconnections, more specifically, this delay should be on the same order of magnitude as the intrinsic timescale of the neuron. In the case of an excitable microring, this timescale is determined by the thermal relaxation time $\tau_{t h} \sim 65 \mathrm{~ns}$. If one would use the time-of-flight through the waveguides as in [6], one would need to use, e.g., $\sim 1-10 \mathrm{~m}$ long low-loss waveguide spirals, resulting in an extremely large footprint, and an additional requirement for optical amplifiers to compensate for the waveguide losses. Even other more advanced approaches to create delay on a chip, using, e.g., Coupled Resonator Optical Waveguides (CROWs) or All-Pass (AP) filters do not reach this slow timescale [7], [8].

Alternatively, one could envisage considering a faster SNN implementation. Recently, in simulation, a network of Vertical-Cavity Surface-Emitting Lasers (VCSEL) was proposed as another optical SNN platform, of which the output was coupled out of the chip towards an optical fiber circuit containing tunable delay lines [9]. Although the excitability timescale of the VCSEL is only $1 \mathrm{~ns}$, resulting in a less stringent demand for long delays in the connections, even this approach is rather bulky, as it is still not fully integrated.

In this paper, we will propose a technique to delay pulses between excitable SOI microrings, that compares in the MHz range favorably to more regular approaches. We will first explain the origin of the delay mechanism, and subsequently design a circuit that fully exhibits this mechanism. Additionally, we will show that the obtained delays are sufficiently large to build recursive optical networks showing hysteresis and hence memory.

\section{SimUlation FRAMEWORK}

The simulations illustrating the concepts of this paper are performed with the nonlinear circuit simulator Caphe [10], [11], using the reduced temporal Coupled Mode Theory (CMT) model and corresponding parameters presented in [4], [5] for a typical SOI $4 \mu \mathrm{m}$ radius symmetric Add-Drop (AD) filter with $540 \mathrm{~nm} \times 220 \mathrm{~nm}$ cross section waveguides, assuming a $3 \mathrm{~dB}$-bandwidth $\lambda_{3 d b}=37 \mathrm{pm}$ and intrinsic $\mathrm{Q}$ of $1.25 \times 10^{5}$. In [4] it was shown that, simulations of this model agree with the experimental results.

\section{DELAY MECHANISM AND CIRCUIT LAYOUT}

The combination of thermal and free-carrier nonlinearities can make microrings class II excitable if they are pumped by a sufficently high power $\mathrm{CW}$ input signal at the blue side of their resonance [5]. As the ring is class II excitable, it can not only be perturbed by short power increases in the input power, but also by power dips. Consequently, as we theoretically showed for two AD filters and experimentally demonstrated using two AP rings in [4], those excitable units can be cascaded, as the subsequent ring reacts to the power dips caused by the preceding ring's excitation. Importantly, the latency of an excitation as a reaction to a power dip is bigger than the latency as a reaction to a power peak. Indeed, when a perturbation excites a ring, in the initial phase of the excitation, both the temperature $\Delta T$ and the number of free carriers $N$ have to raise before the optical output pulse is visible. This explains the higher latency for downward pulses, as this type of input perturbations kicks the ring out of its equilibrium state by decreasing both $\Delta T$ and $N$, such that the ring needs more time to reach the region in phase space with high $\Delta T$ and high $N$, where the optical output pulse is visible. The same mechanism that governs this difference in latency explains why in [4], where two cascaded all-pass rings were perturbed through their common bus, the excitation of the second ring was delayed with respect to the first ring's excitation by the downwards pulse it received from the preceding ring.

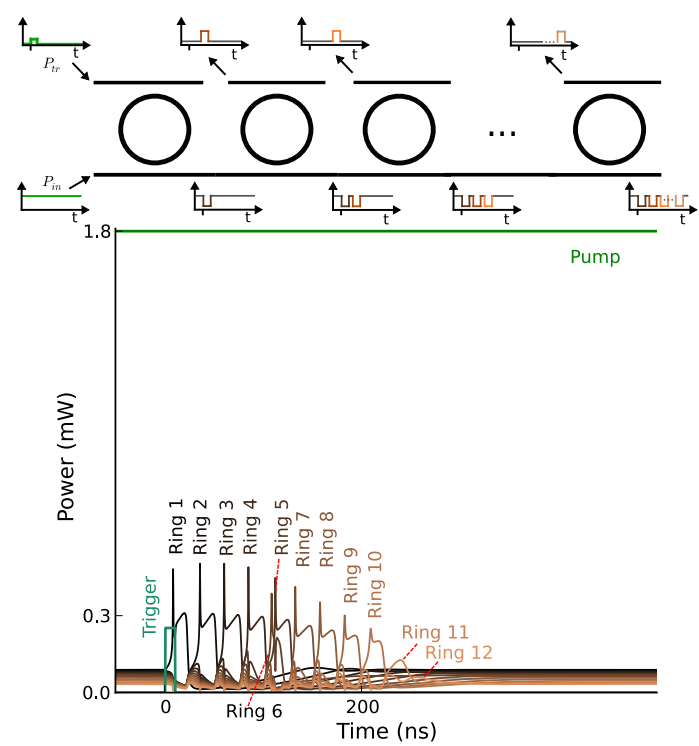

Fig. 1. (top) By cascading a number of excitable add-drop ring resonators on a common bus waveguide, a tapped delay line can be created. (bottom) When pumping a chain of 12 identical rings with a $1.8 \mathrm{~mW} \mathbf{C W}$ signal with a $-16.5 \mathrm{pm}$ offset from resonance, and perturbing it with a $10 \mathrm{~ns}$ long and $0.25 \mathrm{~mW}$ strong trigger pulse, a clear output pulse is retreived at the $10^{t h}$ ring with a $\sim 200 \mathrm{~ns}$ delay. Simulated using Caphe [10], [11].

This higher latency is on the same order of magnitude as the pulse 
width of the excitation. As the resulting delay naturally corresponds to the intrinsic timescale of the neuron, it seems suitable to exploit it as a connection delay mechanism. We do this by cascading several excitable AD filters on a common bus, so that we can make a tapped delay line for an input spike, where the drop ports of the rings contain output pulses that are incrementally delayed with respect to the preceding ring (Fig. 1). Indeed, during an excitation of an $\mathrm{AD}$ filter its resonance temporally changes, such that its transmission drops on the common bus waveguide, increasing the latency of the subsequent rings, while its transmission simultaneously increases at the drop port. Currently, we only need to use a single pump signal for all the rings. This avoids the need to use power splitters, but has as a consequence that every subsequent ring receives less input power than the preceding one, bringing the ring to a power region where it is less excitable and has a smaller output pulse amplitude. Hence, if the ring chain is too long, the last rings will not be excitable anymore or its output pulse might not be strong enough to excite another ring without additional amplification. Furthermore, the signal at the drop port of each ring in the chain contains a ripple corresponding to the fluctuations at the input of the ring due to excitations of the preceding rings. Indeed, while the effective ring resonance changes during an excitation of the ring, resulting in a drastic change in ring output, the transmission change before this optically visible part of the excitation is rather slow, such that the input of the ring is almost linearly transmitted to the drop port, resulting in an attenuated version of the signal leaving the pass port of the previous ring. Accordingly, when using this delay line in a larger network, the coupling sections should be optimized such that the amplitude of this ripple is smaller than the threshold of subsequent optical neurons, while the output pulse is still above threshold.

In between the input excitations of the preceding rings, the ring has time to increase its own $\Delta T$ and $N$, building up its own excitation, while every time it receives a new input spike, its state is set temporally back to a lower $\Delta T$ and $N$ region by the corresponding dip in the input power. The precise timing of its own excitation depends on the complex pattern of the pulse widths, size, and the temporal distance between the preceding pulses. Although in most cases this complex pattern results in an increasing effective delay for every additional ring, unfortunately, sometimes a ring fires simultaneously with its preceding ring. Although this makes it difficult to predict the total effective delay of the chain without detailed simulation, the easy rule of thumb that every additional ring corresponds on the average to an incremental delay in the order of one pulse width holds rather well. Indeed, in our simulation of a chain of twelve rings, the excitation of the $10^{\text {th }}$ ring has a usable output pulse signal with an effective delay of $\sim 200 \mathrm{~ns}$, while the average pulse width $T_{\text {width }}$ is $\sim 15-20 \mathrm{~ns}$.

\section{CLOCK IN MHz RANGE}

To show that the output of this type of delay line is usable to excite subsequent optical neurons, we couple the last output of a delay line of eight rings through a waveguide back to the drop port of the second ring of this chain, such that it is able to excite the counter-clockwise (CCW) mode of this ring (Fig. 2). Initially, after externally perturbing the $\mathrm{CCW}$ mode of the first ring, the whole chain is perturbed, including this $2^{\text {nd }}$ ring. After an excitation, a ring first needs to cool down, before the resonance is again sufficiently close to the input wavelength, to be sensitive to perturbations on this input signal. The time during which the ring recovers from an excitation and is barely sensitive to new perturbations is called the refractory period $T_{\text {refr }}$, of which the exact value depends both on the pump signal and trigger pulse setting, but can be assumed to be in the order of 1-2 $\times \tau_{t h}$ [4]. Consequently, to allow the $8^{\text {th }}$ ring to excite this $2^{\text {nd }}$ ring a second time after its first excitation, the additional delay created by the last part of the delay line should be larger than $T_{r e f r}$ of the microring. This condition is satisfied for the 8 ring chain, such that the excitation of the $2^{\text {nd }}$ ring retriggers the subsequent rings of the chain, inducing a self-sustained oscillation, in which an excitation makes roundtrips

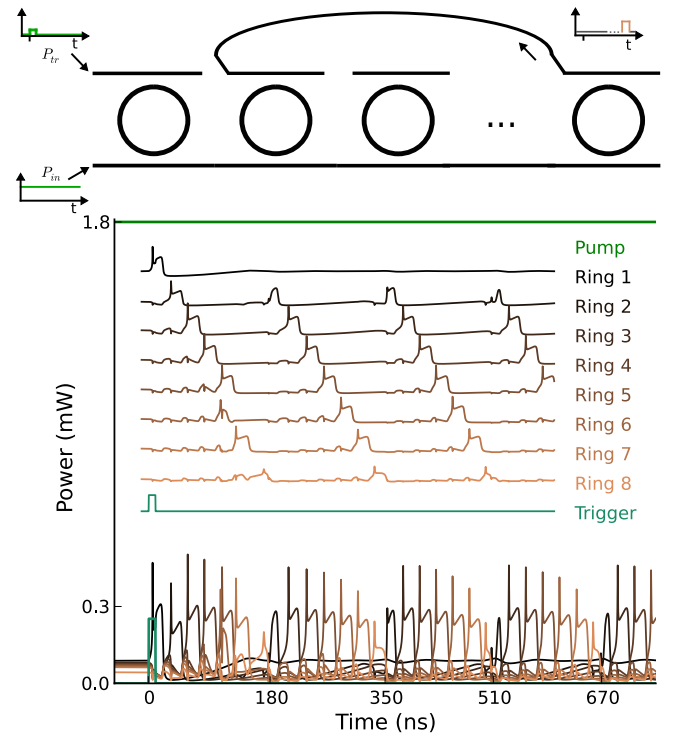

Fig. 2. (top) When feeding back the final output of a tapped optical spike delay line back to one of the inputs of the former rings, this will re-excite the chain and, consequently, an all-optical spike clock can be created. (bottom) We illustrate this for a chain of 8 rings, using the same pumping and trigger conditions as Fig. 1.

through this part of the chain. After an initial transient, the period of this oscillation converges to $\sim 160 \mathrm{~ns}$ and the output at the drop ports of the chain becomes an all-optical clock in the $\mathrm{MHz}$ range. Moreover, as we can switch this chain with an external trigger from its rest state to this oscillating regime, we created a multistable system, demonstrating the capability of our integrated microring platform to retain information during computations [9]

\section{CONCLUSION}

We proposed a passive microring circuit with a small footprint that allows for a delayed transfer of spikes between optical neurons. Consequently, this is a basic building block that is not only needed to design fully integrated optical neural networks, but can also be used in other applications, as there are no suitable integrated circuits that have delays $>100 \mathrm{~ns}$.

\section{ACKNOWLEDGMENT}

This work is supported by IAP Photonics@be of the Belgian Science Policy Office, the Human Brain Project and the ERC NaResCo Starting grant. T. V. V. is supported by the Flemish Research Foundation (FWO-Vlaanderen).

\section{REFERENCES}

[1] A. M. Yacomotti et al., Phys. Rev. Lett. 97, 143904 (2006).

[2] M. Brunstein et al., Phys. Rev. A 85, 031803 (2012).

[3] W. Coomans et al., Phys. Rev. E 84, 1-8 (2011).

[4] T. Van Vaerenbergh et al., Opt. Express 20, 20292-20308 (2012).

[5] T. Van Vaerenbergh et al., Phys. Rev. A 86, 1-8 (2012).

[6] Z. Xiao et al., Opt. Express 21, 21285 (2013).

[7] A. Melloni et al., IEEE Photonics Journal 2, 181-194 (2010).

[8] F. Xia et al., Nat. Photonics 1, 65-71 (2007).

[9] M. A. Nahmias et al., IEEE J. Sel. Top. Quant. Electron. 19, (2013).

[10] M. Fiers et al., J. Opt. Soc. Am. B 29, 896-900 (2011).

[11] www.caphesim.com. 